\title{
Ensino de Bioética e Cuidados Paliativos nas Escolas Médicas do Brasil
}

\author{
The Teaching of Bioethics and Palliative Care \\ in Brazilian Medical Schools
}

José Ricardo de Oliveira ${ }^{I}$ Amauri Carlos Ferreira ${ }^{I I}$ Nilton Alves de Rezende ${ }^{I}$

\section{PALAVRAS-CHAVE: \\ - Bioética; \\ - Cuidados Paliativos; \\ - Educação Médica; \\ - Ensino.}

KEYWORDS:

- Bioethics;

- Palliative Care;

- Medical Education;

- Teaching.
Recebido em: 26/01/2013

Reencaminhado em: 12/04/2013

Reencaminhado em: 26/04/2013

Aprovado em: 02/05/2013

REVISTA BRASILEIRA DE EDUCAÇÃO MÉDICA $285 \frac{27(2): 285-290 ; 2013}{3}$
'Universidade Federal de Minas Gerais, Belo Horizonte, MG, Brasil.

II Pontifícia Universidade Católica de Minas Gerais, Belo Horizonte, MG, Brasil. 


\section{INTRODUÇÃO}

Estudos sobre bioética e terminalidade da vida humana contemplam temas como cuidados paliativos, equipe interdisciplinar de saúde, eutanásia, distanásia e ortotanásia. A atenção aos pacientes sem perspectiva terapêutica convencional ${ }^{1}$ chamados "pacientes terminais" - estabelece o vínculo de cuidado dos membros da equipe interdisciplinar de saúde com pacientes, familiares e cuidadores. Essa relação circunscreve um campo de experiência profissional do cotidiano dos profissionais em todos os níveis de atenção à saúde. Todavia, os responsáveis pela elaboração dos currículos nas Escolas Médicas do Brasil (EMB) parecem não se preocupar com a formação de profissionais que atendam às necessidades emergentes do campo da Bioética, com ênfase em cuidados paliativos. Este aspecto é relevante se se considerar que o Brasil está em processo de transição demográfica, entrando na fase das doenças crônico-degenerativas, em que impera a limitação para as atividades da vida diária e a qualidade de morte.

Embora haja rica literatura e uma práxis sedimentada, internacionalmente, sobre a Bioética e os Cuidados Paliativos, convive-se com dilemas recorrentes no século XXI, que autorizam apresentar algumas indagações:

- Qual o motivo da ausência de uma disciplina curricular, obrigatória ou optativa, relacionada com a terminalidade da vida humana ou com os cuidados de pacientes sem perspectiva terapêutica convencional?

- Por que não há transparência nas atuais propostas pedagógicas, especificamente na área médica, implantando disciplina sobre a temática da terminalidade humana?

- O motivo de ausência e não transparência nessas questões se relacionaria a certo temor desses profissionais de saúde de refletir e discutir sobre a terminalidade da vida humana individual e/ou coletivamente?

$\mathrm{Na}$ tentativa de responder a estes questionamentos, esta revisão foi segmentada em quatro etapas: Bioética Clínica e Cuidados Paliativos; tendências de inserção de Bioética e Cuidados Paliativos nas EMB; a equipe interdisciplinar de Cuidados Paliativos; tentativa de resposta.

\section{BIOÉTICA CLÍNICA E CUIDADOS PALIATIVOS}

Pode-se indagar sobre a contribuição da educação e da formação médicas nas EMB no que concerne à terminalidade da vida humana, com ênfase em Bioética Clínica e Cuidados Paliativos. Para Kovács, a Bioética do século XXI deve retomar a discussão dessas questões relativas ao processo do morrer, da morte e do desenvolvimento humano ${ }^{2}$.

Mas o que é Bioética e Bioética Clínica? Ao lado dos notáveis avanços técnicos e biotecnológicos, surge a Bioética no início dos anos 1960 como experiência de alguns centros acadêmicos, como área de reflexão, discussão e ação. Marca um lugar de encontro interdisciplinar entre profissionais de diversas áreas do conhecimento. No Brasil, as discussões sobre a Bioética, "que poderíamos imprecisamente situar no período de redemocratização do país"3 (p. 28), trouxeram um debate atual sobre avanços da genética, transplante de órgãos, aborto, autonomia dos pacientes, eutanásia e ortotanásia. Portanto, uma discussão densa sobre esses temas auxiliaria pacientes e/ ou familiares na escolha e tomada de decisões. Uma área de reflexão sobre a moral social e os valores pessoais iniciaria a interlocução com a Bioética Clínica? De fato, a Bioética é a ética aplicada às situações de vida cotidiana. A Bioética Clínica adquire densidade nos comitês de Bioética de hospitais universitários e de ética em pesquisas que envolvam seres humanos e animais. A opinião pública, a ética, a ciência e a política estão cada vez mais engajadas em refletir sobre esses temas contemporâneos, considerando que a Bioética cuida da dignidade e da vida, especificamente do significado e do sentido da existência humana ${ }^{1}$.

Acrescenta-se que um número expressivo de estudiosos referencia a Bioética aos dilemas acerca da vida e da morte ${ }^{1,2,4,5}$. Um percurso histórico contempla a morte em distintos cenários da civilização, com suas práticas, ritos e mitos. Transformações sociais provocam mudanças no ambiente onde a morte ocorre. Na Idade Média, a morte acontecia nos domicílios. Nos dias atuais, a morte é relegada ao hospital e, com frequência, confinada aos centros de tratamento intensivo, denominados "catedrais contemporâneas da saúde e do sofrimento humanos" ${ }^{\prime \prime}$ (p. 12).

Níveis de sofisticação tecnológica contribuem não só para a melhoria da expectativa de vida como para a construção de uma cultura que envolve o processo de morte. Uma nova prática, denominada cuidados paliativos, emerge a partir da segunda metade do século XX. Mas o que são os Cuidados Paliativos (CP)? O que significam? A grande maioria dos profissionais da saúde ainda desconhece o que significam e nem sequer sabem da existência de programas e serviços de $\mathrm{CP}$ em instituições de saúde no País ${ }^{5}$. A Organização Mundial da Saúde (OMS) conceituou CP como o cuidado ativo e total aos pacientes cuja doença não responde mais ao tratamento curativo, como: câncer, síndrome da imunodeficiência adquirida, doenças neurológicas, insuficiência hepática e/ou renal, fase final de doenças respiratórias e cardíacas. É importante que o médico reconheça a necessidade da mudança do enfoque terapêutico. A atuação profissional buscaria aliviar o sofrimento por meio do diagnóstico precoce, de uma avaliação minuciosa e do tratamento da dor e de outros sintomas, sejam de natureza física, psicossocial ou espiritual. 


\section{TENDÊNCIAS DE INSERÇÃO DE BIOÉTICA E CUIDADOS PALIATIVOS NAS BEM}

Diante de um enfermo, portador de doença em fase terminal, a OMS e o Conselho Federal de Medicina (CFM), dentre outras entidades, preconizam que sejam adotados os cuidados paliativos, ou seja, uma abordagem voltada para a melhor qualidade de vida tanto dos pacientes quanto de seus familiares e cuidadores ${ }^{1}$.

Com o objetivo de conhecer a realidade do ensino de Ética nos cursos de graduação em Medicina, foram realizadas pesquisas em $1981^{6}, 1984^{6}, 1992^{7}$ e $2001^{8}$.

Ao se fazer uma análise comparativa entre os resultados das últimas duas enquetes realizadas (1992 e 2001), de forma a identificar tendências curriculares inovadoras no campo da Ética, da Deontologia e da Bioética, conclui-se que:

- a variação do número de EMB foi de 76 para 103;

- houve progressão de $96,6 \%$ para $100 \%$ da disciplina de Ética Médica no currículo da graduação;

- em 1992, 33,7\% das Faculdades de Medicina (FM) ministravam a Ética como disciplina autônoma, sendo que $68 \%$ delas a denominavam Deontologia, e em nenhuma o nome era Bioética; após uma década, o número de escolas em que a Ética era disciplina independente aumentou $4 \%$, o termo Deontologia foi mantido por $40 \%$ delas, e em $26,7 \%$ a designação utilizada era Bioética;

- a carga horária da disciplina de Ética se concentrava do terceiro ao quinto ano e na faixa de 10 a 40 horas, resultados obtidos nas duas pesquisas;

- não houve mudanças significativas no ensino da Ética nas FM do Brasil, mas nota-se uma tendência de melhoria ${ }^{8}$.

Recente estudo regional avaliou o desempenho de alunos de Medicina, selecionados em 23 FM do estado de São Paulo, frente às questões paradigmáticas no campo da Bioética. Apesar da elevada presença curricular da Bioética (92,7\%), a ênfase em métodos teóricos de ensino (72,3\%) poderia ser fator desestimulante do aprendizado acadêmico 9 . Alguns estudos exemplares descreveram trajetórias de construção das disciplinas de Bioética/Deontologia e Bioética, respectivamente, em uma FM na cidade de São Paulo (SP) ${ }^{10}$ e na FM da UEL (PR $)^{11}$.

Vale ressaltar as propostas inovadoras de integração curricular - temática, metodológica, transversal, do ensino de Ética Médica e Bioética - na FM-Univali (SC), com disciplina própria do primeiro ao oitavo período, integralizando 135 horas $^{12}$. No mesmo sentido, há a experiência de integração das disciplinas de humanidades médicas da FM-USP (SP), com base humanística, Psicologia Médica, cidadania, Bioética e Bioética Clínica, do primeiro ao quinto ano, com carga horária total de 240 horas $^{13}$
Novas investigações no campo da Bioética contribuem para ampliar conhecimentos sobre a realidade do ensino médico. Figueiredo et al. ${ }^{14}$ constataram que a inclusão da Bioética como disciplina acadêmica se restringia a menos de 30\% dos cursos de graduação e de pós-graduação na área da saúde. Já Dantas e Sousa ${ }^{6}$ afirmaram que não ocorreu alteração significativa na oferta das disciplinas exclusivas de Ética Médica e Bioética. Serodio e Almeida ${ }^{15}$ aprofundaram a pesquisa: entrevistaram profissionais que exerciam atividades de ensino com estudantes de Medicina na Unifesp-EPM (SP) e verificaram algumas discrepâncias em face dos temas abordados em cursos formais de Ética das EMB.

Por fim, citamos as estratégias, ora pontuais, mas bem-sucedidas, do ensino de Bioética nas EMB: Siqueira et al. (UEL-SC) $)^{11}$, Grisard (Univali-SC) ${ }^{12}$, Rios et al. (FM-USP) ${ }^{13}$, Gomes et al. $(\mathrm{UFC}-\mathrm{CE})^{16}$, Drumond et al. (Unimontes-MG) ${ }^{17}$. Apesar desses atuais e exemplares trabalhos na área de Bioética, constata-se escassa referência na literatura nacional quanto ao ensino de cuidados paliativos.

O estudo de Toledo e Priolli ${ }^{18}$ descreveu atitudes e práticas de ensino dos cuidados paliativos no Brasil, conforme relato de 58 coordenadores das EMB participantes da pesquisa (32,4\% de 179 coordenadores). Constatou-se que "a maioria $(96,6 \%)$ considerou muito importante o ensino dos cuidados no fim da vida" (p. 109); entretanto, $73 \%$ acreditam que a carga horária é insuficiente. Embora os coordenadores confirmem sua importância, "ainda é dada pouca prioridade ao ensino deste tema no Brasil" (p. 109)

O relato ${ }^{19}$ contundente de que "Não existe ensino de cuidados paliativos no Brasil. Médicos brasileiros não são treinados para lidar com pacientes portadores de doenças terminais" (p. 19, tradução do autor) enfatiza o olhar pedagógico descompromissado das EMB. As iniciativas, ora pontuais, mas exitosas, no ensino de cuidados paliativos nas EMB seriam: Pinheiro et al. (FM ABC-SP) ${ }^{19}$, Fripp et al. (UFP-RS) ${ }^{20}$, Figueiredo (Unifesp-SP) ${ }^{21}$, Figueiredo e Figueiredo (FMIt-MG) ${ }^{22}$, entre outras.

O estudante de Medicina, ao longo de sua formação, recebe treinamento para lidar com situações cotidianas e mesmo conflituosas, em modalidade curricular real e oculta. Estudos qualitativos exploratórios foram propostos para avaliar o efeito da prática no campo de estágio em dois hospitais-escolas. No hospital universitário da FM-Uerj (RJ), pesquisadores ${ }^{23}$ avaliaram 128 estudantes do internato, enfatizando a perspectiva da percepção dos mesmos quanto aos conflitos e dilemas morais, e confirmaram um grau de defasagem no conteúdo curricular e nas estratégias pedagógicas. Do mesmo modo, no Hospital das Clínicas da UFRS (RS), estudiosos ${ }^{24}$ entrevista- 
ram cinco alunos do estágio prático do internato quanto ao "sentir-se preparado frente à morte e ao morrer" (p. 39) e identificaram uma lacuna na formação acadêmica.

A criação de Ligas Acadêmicas de Medicina Paliativa nas EMB ou grupos de $\mathrm{CP}$ em hospitais-escolas configura a grade do currículo oculto ou paralelo. Também possibilita uma complementação de ensino-aprendizagem e experiência clínica interdisciplinar com certo número de alunos ${ }^{21,22,25,26}$.

\section{A EQUIPE INTERDISCIPLINAR DE CUIDADOS PALIATIVOS}

Uma nova forma de cuidado circunscreve as relações entre paciente, família, cuidador, médico e outros profissionais de saúde. Quais seriam as atitudes dessas pessoas frente ao processo do morrer e da morte?

$\mathrm{O}$ processo da terminalidade humana não deveria ser um problema na área da medicina. É um evento natural, tanto quanto o nascimento, o crescimento e o desenvolvimento. Caberia aos profissionais e aos serviços de saúde orientar, prevenir complicações, assistir e tratar, sempre que possível. A atitude de cuidar de outro que se encontra frágil demarca uma implicação e faz parte de uma ação advinda de escolha pessoal e/ou profissional. No processo do morrer e da morte, o paciente precisa de solidariedade e de reconhecimento de sua dignidade ${ }^{1,2,5}$. Todavia, é necessário que o ensino médico se volte também para despertar a sensibilidade de educadores e educandos no que diz respeito à humanidade dos que estão em situação de limite entre a vida e a morte. Portanto, é pertinente pensar na introdução de temas sobre a terminalidade humana na formação interdisciplinar dos médicos.

A atenção interdisciplinar em CP é realizada por uma equipe específica - composta por médico, enfermeiro, psicólogo, assistente social e outros profissionais necessários ao cuidado -, que entende a importância de cuidar do paciente na mesma dimensão em que cuida da família e da comunida$\mathrm{de}^{1,2,5}$. Quanto à formação de profissionais de saúde e de serviços em CP, Floriani ${ }^{27}$ informa que a expansão global do moderno movimento hospice tem sido rápida e impressionante. Investigações recentes informam que esse movimento é consistente em 115 países, enquanto em cerca de 40 outros países existem condições para seu desenvolvimento, assim como no Brasil. No entanto, vários fatores concorrem para tornar menos eficazes as ações de CP. Faz-se referência à falta de qualificação de recursos humanos (ensino médico) e à existência de políticas de restrita liberação de opioides ${ }^{27}$.

O Ministério da Saúde aprovou a Política Nacional de Atenção Oncológica e de Assistência à Dor, Portaria nº 19/GM (03/01/2002) - nomeada Programa Nacional de Assistência à
Dor e $\mathrm{CP}$-, que cria, no âmbito do SUS, a organização dos $\mathrm{CP}$, com destaque para a necessidade de elaboração do ensino de CP na graduação e na pós-graduação. Entretanto, esta diretriz não efetivou ainda medidas necessárias às mudanças preconizadas. No Brasil ${ }^{28}$, em 2006, faleceram 1.031.691 pessoas. Apenas os óbitos decorrentes de doenças de evolução crônica ou degenerativa e neoplasias corresponderam a mais de $725 \mathrm{mil}$ brasileiros, que faleceram com grande possibilidade de sofrimento intenso por falta de formação, educação continuada e recursos humanos direcionados aos $\mathrm{CP}$.

De outro modo, como parte do processo de edição do novo Código de Ética Médica (2009/2010), a Comissão Mista de Especialidade, formada por representantes do CFM, da Associação Médica Brasileira e da Comissão Nacional de Residência Médica do Ministério da Educação e Cultura (MEC), emitiu parecer favorável à criação da área de atuação em Medicina Paliativa. Indaga-se: como se cria uma subespecialidade médica sem se preocupar com a formação acadêmica? Então, o que muda? Com a regulamentação da Medicina Paliativa, preveem-se mudanças socioculturais e na qualidade do atendimento aos pacientes portadores de doenças crônicas, incuráveis ou que estejam em fase final de vida. A exigência de formação específica, portanto, demanda a criação de cursos de residência médica em Medicina Paliativa. Mas quais seriam os elementos facilitadores para que os egressos das EMB procurassem essa nova área de atuação?

\section{TENTATIVA DE RESPOSTA}

Esta revisão articula dois campos de conhecimento - o da Bioética e o dos Cuidados Paliativos - demarcando a questão inicial: que lugares esses saberes ocupam nos cursos de Medicina autorizados pelo MEC? Nesse contexto, as Diretrizes Curriculares Nacionais ${ }^{29}$ para os cursos de graduação em Medicina contemplam seis competências e habilidades gerais: atenção à saúde, tomada de decisões, comunicação, liderança, gestão em saúde, educação permanente. $\mathrm{O}$ registro da ética e da equipe multiprofissional está presente em partes do texto: [...] profissional humanista, crítico e reflexivo [...] pautado em princípios éticos, legais; [...] devem realizar seus serviços dentro dos mais altos padrões de qualidade e dos princípios da ética/Bioética [...]; [...] compreensão dos processos fisiológicos dos seres humanos - gestação, nascimento, crescimento e desenvolvimento, envelhecimento e do processo de morte [...]; atuar em equipe multiprofissional ${ }^{29}$ (p. 38).

Descortinam-se possibilidades para avaliar os espaços político, teórico e prático/pedagógico no ensino médico quanto ao processo da terminalidade humana. As tendências de mudanças na formação médica, saindo da pauta da agenda de 
saúde para a consolidação da equipe interdisciplinar de Cuidados Paliativos, é um longo percurso de aprendizado, erros e acertos por parte do governo e dos responsáveis pela formação de um(a) competente médico(a) brasileiro(a).

$\mathrm{O}$ cuidado com pessoas em situações de terminalidade reivindica do profissional médico, além de competência técnico-científica, um perfil humanista. A atitude de cuidado cuidar do outro - no campo da saúde é um dos pressupostos da dignidade humana. A reflexão Bioética enriquece "o fazer médico e o saber médico com a oportunidade de termos, na mesma discussão, a presença de juristas, filósofos, sociólogos e outros profissionais" ${ }^{\prime \prime 30}$ (p. 51)

A escassez bibliográfica, as limitações estruturais dos estudos, a falta de um cadastro nacional das EMB, entre nós, dificultam a pesquisa, uma vez que a articulação entre o ensino de Bioética e Cuidados Paliativos tem sido pouco investigada na área da saúde. Como estaria integrado esse tema no currículo das atuais $197 \mathrm{EMB}$ ?

\section{CONCLUSÃO}

O processo de integração das disciplinas de Bioética e de Deontologia Médica encontra-se em expansão curricular na área da saúde. Todavia, o ensino de Bioética e Cuidados Paliativos nas EMB tem merecido pouca atenção dos pesquisadores brasileiros. Embora haja rica literatura e uma práxis sedimentada, internacionalmente, sobre a Bioética e os Cuidados Paliativos, convive-se com dilemas recorrentes no século XXI. Constata-se uma ausência de disciplina, obrigatória ou optativa, de Cuidados Paliativos nas EMB. A ausência dessa disciplina poderia ser creditada, quem sabe, ao desinteresse dos responsáveis pela elaboração curricular. Assim, a proposta de se estudar e responder a essa condição curricular de ensino nas EMB é uma tarefa inicial, mas requer ousadia, podendo ser tema matriz de pesquisas futuras no campo do ensino médico.

\section{REFERÊNCIAS}

1. Oliveira JR. Bioética e atenção ao paciente sem perspectiva terapêutica convencional: estudo sobre o morrer com dignidade. 2007; Belo Horizonte. Mestrado [Dissertação] — Faculdade de Medicina, Universidade Federal de Minas Gerais.

2. Kovács MJ. Bioética nas questões da vida e da Morte. In: Kovács MJ. Educação para a morte: temas e reflexões. São Paulo: Casa do Psicólogo/FAPESP; 2004.

3. Rego S. Reflexão sobre o processo de formação ética dos médicos. Cad ABEM. 2004;1:28-9.

4. Berlinguer G. Bioética cotidiana. Brasília, DF: Universidade de Brasília; 2004.
5. Pessini L, Bertachini L, organizadores. Humanização e cuidados paliativos. 2ª ed. São Paulo: Loyola; 2004.

6. Dantas F, Sousa EG. Ensino da Deontologia, Ética Médica e Bioética nas Escolas Médicas Brasileiras: uma revisão sistemática. Rev Bras Educ Med. 2008;32(4):507-17.

7. Meira AR, Cunha MMS. O ensino da ética médica em nível de graduação nas faculdades de Medicina do Brasil. Rev Bras Educ Med. 1994;18 (1):7-10.

8. Muñoz D, Muñoz DR. O ensino da ética nas Faculdades de Medicina do Brasil. Rev Bras Educ Med. 2003;27:114-24.

9. Oliveira GB, Guaiumi TJ, Cipullo JP. Avaliação do ensino de Bioética nas faculdades de medicina do estado de São Paulo. Arq Ciênc Saúde. 2008;15(3):125-31.

10. Caramico HJ, Zaher VL, Rosito MMB. Ensino da Bioética nas faculdades de medicina do Brasil. Bioethikos. 2007;1(1):76-90.

11. Siqueira JE, Sakai MH, Eisele RL O Ensino da ética no curso de Medicina: a experiência da Universidade Estadual de Londrina (UEL). Simpósio. Bioética. 2002;10(1):86-95.

12. Grisard N. Ética médica e Bioética: a disciplina em falta na graduação médica. Bioética. 2002;10:97-114.

13. Rios IC, Lopes Junior A, Kaufman A, Vieira JE, Scanavino MT, Oliveira RA. A integração das disciplinas de humanidades médicas na faculdade de medicina da USP - Um caminho para o ensino. Rev Bras Educ Med. 2008;32(1):112-21.

14. Figueiredo AM, Garrafa V, Portillo JAC. Ensino da Bioética na área das ciências da saúde no Brasil: estudo de revisão sistemática. R Inter Interdisc INTERthesis. 2008; 5(2):47-72.

15. Serodio AMB, Almeida JAM. Situações de conflitos éticos relevantes para a discussão com estudantes de Medicina: uma visão docente. Rev Bras Educ Med. 2009;33(1):55-62.

16. Gomes AMA, Moura ERF, Amorim RF. O lugar da ética e Bioética nos currículos de formação médica. Rev Bras Educ Med. 2006;30(2):56-65.

17. Drumond JGF, Canela JR, Dias OV, Costa SM. Bioética instrumento de "integração" interdisciplinar e transdisciplinar na UNIMONTES. Anais do III Congresso Brasileiro de Bioética e I Congresso de Bioética do Cone Sul; 3-4 jul. 2000; Porto Alegre, RS: Sociedade Sul-Riograndense de Bioética [online]. 2000 [acesso em 12 dez. 2012]. Disponível em: http:/ / www.bioetica.ufrgs.br/ conres.htm\#16.

18. Toledo AP, Priolli DG. Cuidados no Fim da Vida: O Ensino Médico no Brasil. Rev Bras Educ Med. 2012;36(1):109-117. 
19. Pinheiro T, Blasco P, Benedetto MA, Levites M, Del Giglio A, Mônaco C. Teaching palliative care in a free clinic: a Brazilian experience [online]. [acesso em $12 \mathrm{dez}$. 2012]. Disponível em: http://cdn.intechopen.com/pdfs/27611/ InTech-Teaching_palliative_care_in_a_free_clinic_a_ brazilian_experience.pdf.

20. Fripp JC, Facchini LA, Silva SM. Caracterização de um programa de internação domiciliar e cuidados paliativos no Município de Pelotas, Estado do Rio Grande do Sul, Brasil: uma contribuição à atenção integral aos usuários com câncer no Sistema Único de Saúde, SUS. Epidemiol Serv Saúde. 2012;21(1):69-78.

21. Figueiredo MTA. Prefácio. In: Oliveira RA, coord. Cuidado Paliativo. São Paulo: CREMESP; 2008.

22. Figueiredo MTA, Figueiredo MGMCA. Cuidados Paliativos [online]. Itajubá, MG: Graça Mota Figueiredo. 2010. [acesso em 18 jan. 2013]. Disponível em: http://bilhetedeplataforma.blogspot.com.br/2010/05/texto-nossa-vida-dentro-de-nos.html.

23. Taquette SR, Rego S, Schramm FR, Soares LL, Carvalho SV. Situações eticamente conflituosas vivenciadas por estudantes de medicina. Rev Assoc Med Bras. 2005;51(1):23-8.

24. Azeredo NSG, Rocha CF, Carvalho PRA. O enfrentamento da morte e do morrer na formação de acadêmicos de Medicina. Rev Assoc Med Bras. 2011;35(1):37-43.

25. Liga de Cuidados Paliativos de Caxias do Sul [homepage]. Caxias do Sul, RS: Liga de Cuidados Paliativos; c2013 [acesso em 18 jan. 2013]: [6 telas] Disponível em: http:/ / www.ucs.br/ucs/centro/cecs/ligas/paliativos/ capa/inicial.

26. Academia Nacional de Cuidados Paliativos. Anais do IV Congresso Internacional de Cuidados Paliativos. 6-9 out. 2010; São Paulo, Brasil. São Paulo, SP: ANCP; out. 2010. 132p. [acesso em 18 jan. 2013]. Disponível em: http:// www.paliativo.org.br/dl.php?bid=101.

27. Floriani CA. Palliative care in Brazil: a challenge to the Health-Care System. Palliative Care: Research and Treatment. 2008;2:19-24.

28. Brasil. Ministério da Saúde. Departamento de Informática do SUS. Indicadores e Dados Básicos - Brasil - 2006 [online]. Brasília, DF: MS; 2006. [acesso em 21 out. 2010]. Disponível em: http://tabnet.datasus.gov.br/cgi/idb2006/ matriz.htm

29. Brasil. Ministério da Educação. Conselho Nacional de Educação. Câmara de Educação Superior. Resolução CNE-CES n. 4. 2001. Institui as Diretrizes Curriculares Nacionais do Curso de Graduação em Medicina. Diário Oficial da União. 2001 nov. 9; seção 1:38.

30. D'Ávila RL. O Conselho Federal de Medicina e o ensino da Ética e Bioética. Bioética. 2003;11(2):51-6.

\section{CONTRIBUIÇÃO DOS AUTORES}

José Ricardo de Oliveira contribuiu na concepção e desenho do estudo, análise e interpretação dos dados, produção e revisão do texto final. Amauri Carlos Ferreira e Nilton Alves de Rezende participaram da concepção do estudo e revisão do texto final.

\section{CONFLITO DE INTERESSES}

Declarou não haver.

\section{ENDEREÇO PARA CORRESPONDÊNCIA}

José Ricardo de Oliveira

Rua Maracaju, 19 - apto 1603

Nova Granada - Belo Horizonte

CEP 30431-350 - MG

E-mail: oliveira.jricardo@gmail.com 